\title{
Correlation between the Accumulation of Skin Glycosylation End Products and the Development of type 2 Diabetic Peripheral Neuropathy
}

\section{Xing-Wang Zhao}

Jianyang Hospital of Traditional Chinese Medicine

\section{Wan-Xu Yue}

The People's Hospital of Wuhua District

Sen-Wei Zhang

Chengdu University of TCM

Chen Qiu ( $\square$ chenqiu1005@cdutcm.edu.com)

Chengdu University of Traditional Chinese Medicine Affiliated Hospital

\section{Research Article}

Keywords: Advanced glycation end products, Detection method, Illness assessment

Posted Date: September 7th, 2021

DOI: https://doi.org/10.21203/rs.3.rs-776672/v1

License: (c) (i) This work is licensed under a Creative Commons Attribution 4.0 International License. Read Full License 


\section{Abstract}

With the increasing prevalence, disability and mortality of diabetic peripheral neuropathy (DPN), convenient, effective and inexpensive examination methods remain lacking. Advanced glycation end products (AGEs) have been shown to cause DPN independently or indirectly .Presently, the methods of detecting its concentration in serum are complicated and limited. AGE Pro developed by the Chinese Academy of Sciences uses the principle of positive correlation between fluorescence intensity of specific excitation/emission band and AGEs accumulation amount in skin tissues to invert the AGE level of subjects in vivo based on the obtained optical signals, to realize the risk and disease assessment of diabetes complications. Through the detection of skin AGEs with different severity of diabetic peripheral neuropathy and retrospective analysis of relevant data, this study showed that the accumulation of skin AGEs could reflect the severity of DPN to some extent. Skin AGEs were proven to be a good reference for the diagnosis and severity evaluation of DPN.

\section{Introduction}

Diabetic peripheral neuropathy (DPN) is a common chronic complication of diabetes, and its incidence rate is also increasing, which is an important cause of foot ulcer, infection, arterial thrombosis, muscular atrophy, fall fracture, gangrene and even amputation. The ineffective or poor treatment of DPN increases the expenditure of patients with diabetes and also causes the waste of national medical resources. Thus, conducting diabetes screening as early as possible and evaluating the risk value and severity of diabetes and its complications are of great significance.

AGEs are covalent adders with high activity and stability produced by nonenzymatic glycation reactions (also known as Maillard reactions) without the involvement of enzymes. AGEs are highly heterogeneous, with several existing forms in human tissues and organs. Currently recognized structural forms include pentosidine, carboxymethyl lysine, carboxyethyl lysine pyrraline and crossline. AGEs plays an important role in the evolution of diabetes and its complications.

Presently, the pathogenesis of diabetic peripheral neuropathy is not very clear. Clinical and animal data suggest that AGEs induced by hyperglycemia plays an important role in nerve injury ${ }^{[1]}$, which is closely related to other metabolic mechanisms, including increasing its number through the polyol pathway, enhancing oxidative stress ${ }^{[2]}$, and activating the diacylglycerol kinase $C(P K C)$ pathway ${ }^{[3-4]}$.All of these mechanisms contribute to increased oxidative stress, which in turn increases the formation of glycation products, creating a vicious cycle. Other studies ${ }^{[5]}$ have found that diabetic peripheral neuropathy, especially small-fiber neuropathy, is significantly correlated with the increase of AGEs in the skin. Dipino ${ }^{[6]}$ et al. confirmed that a large part of the human body's AGEs is exogenous, which is an important source of the accumulation of AGEs in the body. Glycation toxicity caused by overcooking of animal-derived foods has been reported under different pathological conditions, including chronic kidney disease and aging process. Negrean et al. ${ }^{[7]}$ demonstrated in a systematic analysis of type 2 diabetes patients that 
microvascular function was impaired after high-dose diets, and serum AGEs and oxidative stress markers were elevated.

Although the correlation between serum AGEs and neuropathy has been confirmed, only a few small sample case studies abroad ${ }^{[8-9]}$ have shown is a certain correlation between AGEs measured by skin fluorescence and type 1 diabetic peripheral neuropathy, which is mainly used in Caucasians. In Asian populations, the association between cumulative skin AGEs and DPN of varying severity in type 2 diabetic patients has not been reported in the literature.

\section{Experimental Procedures}

\section{Study participants}

This study included 560 type 2 diabetes hospitalized in the department of endocrinology, affiliated hospital of Chengdu University of Traditional Chinese Medicine from May 2016 to June 2018. The participants included 312 and 248 men and women, respectively. All experiments on human subjects were conducted in accordance with the Declaration of Helsinki and that all procedures were carried out with the adequate understanding and written consent of the subjects.

\section{Inclusion and exclusion criteria}

The inclusion criteria included the following: Patients who meet the diagnostic criteria of T2DM in the WHO criteria for diabetes diagnosis and classification (1999), conform to the diagnostic criteria of DSPN published by ADA in 2010, aged >18years, and participate voluntarily.

Moreover, the exclusion criteria included the following:

(1) Minor patients;

(2) Type 1 and a special type of diabetes;

(3) Recent acute complications of DM, including DKA, severe hypoglycemia coma and co-infected patients;

(4) Patients with severe liver function impairment (AST or ALT 2.5 times higher than the upper limit of normal), including patients with cirrhosis, severe hepatitis or severe cardiovascular and blood diseases;

(5) Neuropathy caused by other factors was diagnosed, including severe macrovascular abnormalities (thrombosis, etc.),cervical and lumbar problems (pinched nerve roots, narrow spinal canal, senile vertebral changes), cerebral infarction, Guillain-Barre syndrome, and neurotoxic and side effects caused by special drugs. The accumulation of toxic substances in the body caused by renal insufficiency causes nerve damage; 
(6) Patients with other endocrine diseases that can significantly increase blood glucose, including hyperthyroidism, cortisolism, Cushing's syndrome, or those who have recently used glucocorticoids;

(7) Patients with mental illness, deformity or other reasons that cannot complete the test with the assistance of the detection personnel;

(8) Patients with a large area of scar, rash, vitiligo, or other infectious skin diseases on the ulnar skin of the left forearm.

After screening, samples are eliminated from the study if patients are not suitable for inclusion or exclusion criteria and incomplete data collection.

The reason for the removal of patient cases should be explained, and the statistical personnel and research team should make a plan to determine whether the case is deleted or not.

\section{Research method}

\section{Detection methods of skin fluorescence AGEs}

Test process: after calibrating the instrument, patients must fill in their basic information. Subjects will be seated at a constant room temperature (approximately $25^{\circ} \mathrm{C}$ ) with their left forearm flat on the arm limit bracket, palms down, and the inner forearm skin (approximately 10-15 cm below the elbow) attached to the scanner probe. The forearm should be raised with the elbow as the fulcrum between each of the three scans until the forearm is removed from the probe and the forearm position is restored. The instrument automatically averages the three results and estimates the risk of four major complications. Note:The skin should not have visible blood vessels, scars, moss-like plaques, vitiligo, malformation, or other skin abnormalities. If the hair is strong, it should be removed and tested. Do not apply care cream or any fluorescent substance, including sunscreen or body lotion, and wash and dry with clean water before testing.

\section{Grouping criteria}

Participants were grouped according to three grouping criteria: P. J. Dyck criteria, full box results, and the Toronto Clinical Scoring System (TCSS).

P. J. Dyck criteria described the severity stages of neuropathy as follows: grade 0 (non-DPN group), diabetic group without neuropathy; grade 1 (early stage), asymptomatic neuropathy; grade 2 (middle stage), neuropathic symptoms are present, but without neurological involvement; and grade 3 (advanced), presence of neuropathic symptoms and functional involvement ${ }^{[10]}$. 
For full box results, vibration perception threshold (VPT) of $<15$ is the low-risk group, $15-25$ is the medium-risk group, and $>25$ is the high-risk group. According to the detection results of the current perception threshold (CPT),it was divided into normal group, attenuated group and sensitive group.

For the TCSS, total scores of $0-5,6-8,9-11$, and 12-19 points are classified as normal, mild, moderate, and severe, respectively ${ }^{[11]}$.

\section{Observational index}

The following data are collected: name, sex, age, course of illness, blood pressure, $\mathrm{BMI}, \mathrm{Ca}^{2+}$, biochemical indicators (UA, Cr, CysC, and Hcy), inflammation index (WBC, NEUT, LY, neutrophil-lymphocyte ratio [NLR], and CRP), renal function index (eGFR, ACR, MAU, a-MG, Tf, and Ig), blood lipid (TC,TG,LDL, and HDL), $\mathrm{HbA1c}$, FPG, 1Hpbg, 2hPBG, 3hPBG, insulin (fasting and postprandial respectively 1, 2, and $3 \mathrm{~h}$ ), C-peptide (fasting and postprandial $2 \mathrm{~h}$ ), HOMA-IR, VPT, CPT,TCSS.

\section{Data statistics and analysis methods}

SPSS 20.0 statistical software was used for data analysis. Statistical charts were made by GraphPad Prism and SPSS 20.0 statistical software. $P<0.05$ and $P<0.01$ were considered statistically significant. $(n=79)$

\section{Research Results}

\section{Correlation of indicators among DPN groups}

\section{Normal data analysis}

Table 1

Comparison of clinical and laboratory indicators between groups $\left(\mathrm{x}^{-} \pm \mathrm{s}\right)$ 


\begin{tabular}{|c|c|c|c|c|c|c|}
\hline Indicators & $\begin{array}{l}\text { Grade } 0 \\
(n=79)\end{array}$ & $\begin{array}{l}\text { Grade } 1 \\
(n=199)\end{array}$ & $\begin{array}{l}\text { Grade } 2 \\
(\mathrm{n}=202)\end{array}$ & $\begin{array}{l}\text { Grade } 3 \\
(n=80)\end{array}$ & $\mathrm{F}$ & $P$ \\
\hline AGEs (AU) & $74.36 \pm 11.97$ & $\begin{array}{l}84.02 \pm \\
13.88\end{array}$ & $\begin{array}{l}87.72 \pm \\
15.67\end{array}$ & $\begin{array}{l}93.99 \pm \\
19.83\end{array}$ & 24.290 & $.000 *$ \\
\hline Course (years) & $2.96 \pm 2.91$ & $7.54 \pm 6.48$ & $10.27 \pm 7.23$ & $8.36 \pm 5.30$ & 4.032 & $.009 *$ \\
\hline Age (years) & $50.2 \pm 12.71$ & $\begin{array}{l}59.31 \pm \\
11.26\end{array}$ & $\begin{array}{l}62.06 \pm \\
11.89\end{array}$ & $\begin{array}{l}69.85 \pm \\
10.87\end{array}$ & 39.423 & $.000 *$ \\
\hline Weight (kg) & $62.91 \pm 12.95$ & $\begin{array}{l}65.22 \pm \\
11.51\end{array}$ & $\begin{array}{l}63.58 \pm \\
11.10\end{array}$ & $\begin{array}{l}62.71 \pm \\
10.31\end{array}$ & 1.420 & .236 \\
\hline BMI (kg/m2) & $24.31 \pm 3.50$ & $24.48 \pm 3.34$ & $24.42 \pm 3.79$ & $23.70 \pm 3.12$ & 1.031 & .378 \\
\hline $\mathrm{Ca}^{2+}(\mathrm{mmol} / \mathrm{L})$ & $2.21 \pm 0.14$ & $2.21 \pm 0.13$ & $2.19 \pm 0.15$ & $2.18 \pm 0.13$ & 1.293 & .276 \\
\hline $\mathrm{UA}(\mu \mathrm{mol} / \mathrm{L})$ & $\begin{array}{l}316.98 \pm \\
100.82\end{array}$ & $\begin{array}{l}336.52 \pm \\
100.30\end{array}$ & $\begin{array}{l}332.97 \pm \\
100.92\end{array}$ & $\begin{array}{l}355.74 \pm \\
90.62\end{array}$ & 2.074 & .103 \\
\hline $\mathrm{TC}(\mathrm{mmol} / \mathrm{L})$ & $4.62 \pm 1.17$ & $4.40 \pm 1.16$ & $4.40 \pm 1.08$ & $4.39 \pm 1.36$ & .815 & .486 \\
\hline LDL (mmol/L) & $2.69 \pm 0.91$ & $2.57 \pm 0.81$ & $2.54 \pm 0.78$ & $2.54 \pm 0.88$ & .617 & .604 \\
\hline WBC (*109/L) & $6.05 \pm 1.83$ & $6.10 \pm 1.93$ & $6.14 \pm 1.89$ & $6.02 \pm 2.17$ & .067 & .978 \\
\hline $\begin{array}{l}\text { 1hPBG } \\
(\mathrm{mmol} / \mathrm{L})\end{array}$ & $14.99 \pm 4.24$ & $14.27 \pm 3.56$ & $15.31 \pm 2.72$ & $15.02 \pm 4.48$ & .506 & .679 \\
\hline $\begin{array}{l}2 \mathrm{hPBG} \\
(\mathrm{mmol} / \mathrm{L})\end{array}$ & $17.71 \pm 5.17$ & $18.07 \pm 4.25$ & $17.29 \pm 4.83$ & $20.85 \pm 6.18$ & 2.453 & .064 \\
\hline $\begin{array}{l}\text { 3hPBG } \\
(\mathrm{mmol} / \mathrm{L})\end{array}$ & $15.97 \pm 6.60$ & $16.30 \pm 4.62$ & $18.52 \pm 3.13$ & $19.15 \pm 4.95$ & 1.716 & .171 \\
\hline INS60'(mIU/L) & $47.41 \pm 54.60$ & $\begin{array}{l}25.75 \pm \\
17.67\end{array}$ & $\begin{array}{l}17.95 \pm \\
10.10\end{array}$ & $13.08 \pm 5.48$ & 4.106 & $.009 *$ \\
\hline INS120'(mIU/L) & $62.25 \pm 70.87$ & $\begin{array}{l}28.90 \pm \\
17.50\end{array}$ & $\begin{array}{l}24.61 \pm \\
14.71\end{array}$ & $15.93 \pm 9.89$ & 4.613 & $.005^{\star}$ \\
\hline INS180'(mIU/L) & $48.10 \pm 54.17$ & $\begin{array}{l}28.66 \pm \\
18.60\end{array}$ & $\begin{array}{l}25.82 \pm \\
15.55\end{array}$ & $19.35 \pm 9.55$ & 2.291 & .085 \\
\hline
\end{tabular}

* The significance level of the mean difference is 0.05 .

Results: (Table 1): One-way analysis of variance (ANOVA) (Table 1) was conducted for the data according to the normal distribution: the significance of age, AGEs, postprandial 1, $2 \mathrm{~h}$ insulin, and course of disease were all $<0.05$, indicating that statistically significant differences were found between the two groups in the indicators mentioned above at least. Among them, the significance of AGEs ,INS60', and INS120' was all $<0.05$, indicating a linear relationship between groups. 
Non-normal measurement data analysis 
Table 2

Comparison of clinical and laboratory indicators between groups (median (lower quartile upper quartile))

\begin{tabular}{|c|c|c|c|c|c|}
\hline Variable & $\begin{array}{l}\text { Grade } 0(n= \\
79)\end{array}$ & $\begin{array}{l}\text { Grade } 1(n= \\
199)\end{array}$ & $\begin{array}{l}\text { Grade } 2(n= \\
202)\end{array}$ & Grade $3(n=80)$ & $\mathrm{P}$ \\
\hline TG囚mmol/L】 & $\begin{array}{l}1.86 \rrbracket 1.13- \\
2.93 \rrbracket^{\mathrm{c} \#}\end{array}$ & $\begin{array}{l}1.74 \rrbracket 1.13- \\
2.71 \rrbracket^{\mathrm{e} \#}\end{array}$ & $\begin{array}{l}1.57 \rrbracket 1.07- \\
2.40 \rrbracket\end{array}$ & $\begin{array}{l}1.385 \rrbracket 0.92- \\
2.12 \rrbracket\end{array}$ & $0.016 *$ \\
\hline 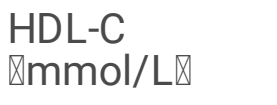 & $\begin{array}{l}0.99 \varangle 0.82- \\
1.16 \rrbracket\end{array}$ & $\begin{array}{l}1.015 \rrbracket 0.84- \\
1.16 \rrbracket\end{array}$ & $\begin{array}{l}1.025 \rrbracket 0.87- \\
1.22 \rrbracket\end{array}$ & $\begin{array}{l}1.07 \rrbracket 0.93- \\
1.32 \rrbracket\end{array}$ & 0.069 \\
\hline 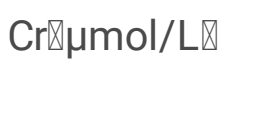 & $\begin{array}{l}59.1 \otimes 48.90- \\
68.20 \rrbracket\end{array}$ & $\begin{array}{l}63.25 \bowtie 54.18- \\
76.98 \rrbracket^{\mathrm{e} \#}\end{array}$ & $\begin{array}{l}61.95 \llbracket 51.40- \\
76.28 \rrbracket^{f \# \#}\end{array}$ & $\begin{array}{l}73.5 \rrbracket 59.53- \\
88.00 \rrbracket^{\mathrm{c} \# \#}\end{array}$ & $0.000 *$ \\
\hline CysCØmg/L】 & $\begin{array}{l}0.78 \rrbracket 0.67- \\
0.89 \rrbracket^{a \# \#}\end{array}$ & $\begin{array}{l}0.935 \bowtie 0.77- \\
1.21 \mathbb{\mathrm { e }}^{\mathrm{A} \#}\end{array}$ & $\begin{array}{l}0.935 \rrbracket 0.80- \\
1.18 \rrbracket^{\mathrm{bf \# \#}}\end{array}$ & $\begin{array}{l}1.09 \rrbracket 0.90- \\
1.47 \rrbracket^{\mathrm{c} \# \#}\end{array}$ & $0.000 *$ \\
\hline Hcy $₫ \mu \mathrm{mol} / \mathrm{L} \nabla$ & $\begin{array}{l}10.03 \rrbracket 7.21- \\
12.47 \rrbracket^{\mathrm{c} \# \#}\end{array}$ & $\begin{array}{l}9.91 \otimes 7.81- \\
13.26 \rrbracket\end{array}$ & $\begin{array}{l}9.77 \rrbracket 8.22- \\
12.88 \rrbracket^{f \# \#}\end{array}$ & $\begin{array}{l}12.1 \otimes 9.55- \\
15.79 \rrbracket^{\mathrm{e} \# \#}\end{array}$ & $0.001 *$ \\
\hline ACR $\rrbracket \mathrm{mg} / \mathrm{g} \rrbracket$ & $\begin{array}{l}12.35 \rrbracket 5.24- \\
33.89 \rrbracket^{\mathrm{c \# \#}}\end{array}$ & $\begin{array}{l}14.46 \rrbracket 5.83- \\
73.43 \rrbracket^{\mathrm{e} \#}\end{array}$ & $\begin{array}{l}16.63 \llbracket 5.62- \\
109.55 \rrbracket^{f \#}\end{array}$ & $\begin{array}{l}51.21 \rrbracket 16.64- \\
296.16 \rrbracket\end{array}$ & $0.005^{\star}$ \\
\hline $\begin{array}{l}\text { eGFR } \\
\bigotimes \mathrm{ml} / \mathrm{min} \rrbracket\end{array}$ & $\begin{array}{l}109.59 \\
\varangle 98.39- \\
120.75 \rrbracket\end{array}$ & 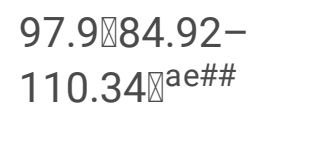 & $\begin{array}{l}96 \rrbracket 80.98- \\
108.94 \rrbracket^{\text {bf\#\# }}\end{array}$ & $\begin{array}{l}84.84 \llbracket 64.43- \\
100.41 \rrbracket^{\mathrm{c} \# \#}\end{array}$ & $0.000 *$ \\
\hline MAU『mg/L】 & $\begin{array}{l}10.6 \rrbracket 5.34- \\
25.60 \rrbracket^{\mathrm{c} \# \#}\end{array}$ & $\begin{array}{l}11.8 \rrbracket 5.15- \\
62.00 \rrbracket^{\mathrm{e} \# \#}\end{array}$ & $\begin{array}{l}12.65 \rrbracket 4.36- \\
51.03 \rrbracket^{f \# \#}\end{array}$ & $\begin{array}{l}47.1 \otimes 9.39- \\
239.50 \rrbracket\end{array}$ & $0.001 *$ \\
\hline a-MG『mg/L】 & $\begin{array}{l}9.96 \rrbracket 55.25- \\
15.73 \rrbracket^{\mathrm{c} \# \#}\end{array}$ & $\begin{array}{l}12.6 \rrbracket 5.30- \\
25.80 \rrbracket\end{array}$ & $\begin{array}{l}10.5 \otimes 5.01- \\
24.10 \rrbracket^{f \#}\end{array}$ & $\begin{array}{l}19.1 \otimes 7.79- \\
34.30 \rrbracket\end{array}$ & $0.004^{*}$ \\
\hline $\mathrm{Tf} \bigotimes \mathrm{mg} / \mathrm{L} \rrbracket$ & $\begin{array}{l}2 \rrbracket 2.00-2.00 \rrbracket \\
\text { c\#\# }\end{array}$ & $2 \varangle 2.00-4.46 \rrbracket^{\mathrm{e} \#}$ & $\begin{array}{l}2 \rrbracket 2.00-2.92 \rrbracket \\
f \# \#\end{array}$ & $\begin{array}{l}2.12 \rrbracket 2.00- \\
14.00 \rrbracket\end{array}$ & $0.000 *$ \\
\hline U-lg G囚mg/L】 & $\begin{array}{l}3.75 \rrbracket 3.00- \\
10.19 \rrbracket^{\mathrm{c} \# \#}\end{array}$ & $\begin{array}{l}5.36 \rrbracket 3.00- \\
13.75 \rrbracket^{\mathrm{e} \#}\end{array}$ & $\begin{array}{l}4.36 \rrbracket 3.00- \\
11.40 \rrbracket^{f \# \#}\end{array}$ & $\begin{array}{l}9.15 \rrbracket 4.03- \\
36.10 \rrbracket\end{array}$ & $0.001 *$ \\
\hline NEUT $\otimes 10^{9} / \mathrm{L} \otimes$ & $\begin{array}{l}3.305 \rrbracket 2.70- \\
4.08 \rrbracket\end{array}$ & $3.5 \rrbracket 2.70-4.34 \rrbracket$ & $\begin{array}{l}3.62 \rrbracket 2.67- \\
4.52 \rrbracket\end{array}$ & $\begin{array}{l}3.74 \rrbracket 2.80- \\
4.60 \rrbracket\end{array}$ & 0.468 \\
\hline LY $\otimes 10^{9} / L \otimes$ & $\begin{array}{l}1.935 \rrbracket 1.45- \\
2.54 \rrbracket\end{array}$ & $\begin{array}{l}1.66 \rrbracket 1.25- \\
2.17 \rrbracket^{a \#}\end{array}$ & $\begin{array}{l}1.69 \rrbracket 1.25- \\
2.04 \rrbracket^{b \#}\end{array}$ & $\begin{array}{l}1.51 \rrbracket 1.26- \\
1.84 \rrbracket^{\mathrm{c} \# \#}\end{array}$ & $0.001^{*}$ \\
\hline NLR & $\begin{array}{l}1.64 \rrbracket 1.40- \\
2.24 \rrbracket\end{array}$ & $\begin{array}{l}2.01 \rrbracket 1.56- \\
2.72 \rrbracket^{a \#}\end{array}$ & $\begin{array}{l}2.13 \rrbracket 1.56- \\
2.86 \rrbracket^{\mathrm{b} \# \#}\end{array}$ & 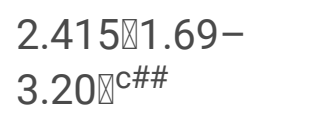 & $0.000 *$ \\
\hline CRP』mg/L】 & $\begin{array}{l}0.7 \rrbracket 0.50- \\
1.80 \rrbracket\end{array}$ & $0.8 \otimes 0.50-2.58 \rrbracket$ & $0.9 \varangle 0.50-2.60 \rrbracket$ & $1 \otimes 0.60-7.60 \rrbracket^{\mathrm{c} \#}$ & $0.033^{*}$ \\
\hline HOMA-IR & $1.86 \otimes 1.37-$ & $1.82 \otimes 1.18-$ & $2.005 \bigotimes 1.28-$ & $0.93 \bowtie 0.52-$ & 0.257 \\
\hline
\end{tabular}




\begin{tabular}{|c|c|c|c|c|c|}
\hline & $6.40 \bigotimes$ & $3.43 \rrbracket$ & $3.07 \rrbracket$ & $1.42 \rrbracket$ & \\
\hline $\begin{array}{l}\text { PFG } \\
\text { 『mmol/L】 }\end{array}$ & $\begin{array}{l}8.47 \rrbracket 6.38- \\
11.35 \rrbracket\end{array}$ & $\begin{array}{l}8.3 \otimes 6.41- \\
10.17 \rrbracket\end{array}$ & $\begin{array}{l}7.94 \rrbracket 5.94- \\
10.53 \rrbracket\end{array}$ & $\begin{array}{l}8.425 \rrbracket 6.25- \\
11.53 \rrbracket\end{array}$ & 0.394 \\
\hline FINS『mIU/L》 & $\begin{array}{l}6.78 \rrbracket 3.85- \\
14.75 \rrbracket\end{array}$ & $\begin{array}{l}7.005 \bowtie 3.36- \\
10.260\end{array}$ & $\begin{array}{l}5.84 \rrbracket 3.93- \\
8.59 \rrbracket\end{array}$ & $\begin{array}{l}3.57 \rrbracket 2.29- \\
4.46 \rrbracket\end{array}$ & 0.302 \\
\hline $\begin{array}{l}\text { C-Peptide } \\
\text { \nmol/L】 }\end{array}$ & $\begin{array}{l}0.666 \rrbracket 0.45- \\
0.83 \rrbracket\end{array}$ & $\begin{array}{l}0.7275 \rrbracket 0.48- \\
0.96 \rrbracket\end{array}$ & $\begin{array}{l}0.646 \rrbracket 0.50- \\
0.87 \rrbracket\end{array}$ & $\begin{array}{l}0.554 \rrbracket 0.42- \\
0.69 \rrbracket\end{array}$ & 0.158 \\
\hline $\begin{array}{l}\text { 2hC-peptide } \\
\text { Ønmol/L】 }\end{array}$ & $\begin{array}{l}1.6 \otimes 1.22- \\
2.47 \rrbracket\end{array}$ & $\begin{array}{l}1.85 \rrbracket 1.29- \\
2.53 \rrbracket\end{array}$ & $\begin{array}{l}1.5981 .10- \\
2.100\end{array}$ & $1.3 \rrbracket 0.79-2.06 \rrbracket$ & 0.142 \\
\hline $\mathrm{HbA1c \unrhd \% \rrbracket}$ & $9 \otimes 7.08-10.48 \rrbracket$ & $\begin{array}{l}8.35 \otimes 6.63- \\
11.08 \rrbracket\end{array}$ & $\begin{array}{l}7.8 \otimes 6.70- \\
10.30 \rrbracket\end{array}$ & $\begin{array}{l}7.3586 .10- \\
9.88 \rrbracket\end{array}$ & 0.103 \\
\hline
\end{tabular}

${ }^{*}$ The progressive significance level was 0.05 . Significance of pared comparison after adjustment $\# \mathrm{P}<0.05$, significance of paired comparison after adjustment; $\# \# \mathrm{P}<0.01 . \mathrm{a}$ : comparison of grades 0 and $1 ;$ b: comparison between grades 0 and $2 ; \mathrm{c}$ : comparison of grades 0 and 3; d: comparison between levels 1 and 2; e: comparison between levels 1 and 3 ; f: comparison of levels 2 and 3.

Results (Table 2): (1). Kruskal-Wallis $\mathrm{H}$ rank and inspection: TG, eGFR, Cr, CysC, Hcy, ACR, MAU, a-MG, Tf, U-Ig G, LY, NLR, and CRP showed significant differences among groups $(P<0.05)$, indicating that statistically significant differences between the two groups were found in the indicators mentioned above at least.

(2) Pairwise comparison after adjustment: $\triangle$ For lipid indicators, TG levels were significantly different between grades 3,0 , and $1(P<0.01$ or $P<0.05)$. . For renal function indicators, significant differences in eGFR were found between grades 01,2 , and 3 and between grades 3,1 , and $2(P<0.01$ or $P<0.05)$.

Significant differences in $\mathrm{Cr}$, ACR, Hcy, MAU, Tf, and Ig, were found between grades $0,1,2$ and $3(P<0.01$ or $P<0.05$ ).Significant differences in CysC were found between gradeS $0,1,2$, and 3 , and between grades 1,2 and $3(P<0.01)$. The difference of $a-M G$ between grades 0,2 , and 3 was found to be significant $(P<$ 0.01 or $\mathrm{P}<0.05)$. $\otimes$ For inflammation-related indicators, a significant difference in CRP was found between grades 0 and $3(P<0.05)$. Significant differences in LY and NLR were found between grades $0,1,2$ and grade $3(P<0.01$ or $P<0.05)$.

\section{Correlation study of AGEs and other indicators}


Table 3

Correlation analysis of AGEs and other indicators by Pearson

\begin{tabular}{|c|c|c|}
\hline Indicators & $r$ & $P$ \\
\hline Age & $.434^{\star \star}$ & .000 \\
\hline BMI & $-.163^{\star \star}$ & .000 \\
\hline $\mathrm{Ca}^{2+}$ & -.001 & .984 \\
\hline UA & $.175^{\star \star}$ & .000 \\
\hline TC & .003 & .937 \\
\hline LDL & .021 & .614 \\
\hline WBC & $.139 \star \star$ & .002 \\
\hline $1 \mathrm{hPBG}$ & .045 & .696 \\
\hline $2 \mathrm{hPBG}$ & .079 & .217 \\
\hline 3hPBG & .164 & .150 \\
\hline INS60' & $-.310 * \star$ & .006 \\
\hline INS120' & $-.302^{\star \star}$ & .008 \\
\hline INS180' & $-.246^{\star}$ & .030 \\
\hline Course & $.312^{\star \star}$ & .001 \\
\hline SBP & $.241^{\star \star}$ & .005 \\
\hline DBP & -.053 & .539 \\
\hline
\end{tabular}

** Significant correlation at 0.01 level; * significantly correlated at the 0.05 level.

Pearson correlation analysis of the cumulative volume of skin AGEs showed that AGEs were positively correlated with age, blood uric acid, white blood cells, disease course and systolic blood pressure. However, it was negatively correlated with BMI and insulin 1-3 $\mathrm{h}$ postprandial (Table 3 ). 
Table 4

Spearman correlation analysis of AGEs and other indicators

\begin{tabular}{|c|c|c|}
\hline Indicators & $r_{s}$ & $P$ \\
\hline CVD risk score & $.831^{\star \star}$ & .000 \\
\hline DPN risk score & $.756 \star \star$ & .000 \\
\hline DR risk score & $.813^{\star \star}$ & .000 \\
\hline DN risk score & $.818^{\star \star}$ & .000 \\
\hline TG & -.040 & .346 \\
\hline HDL-C & .039 & .366 \\
\hline $\mathrm{Cr}$ & $.395^{\star \star}$ & .000 \\
\hline CysC & $.381^{\star *}$ & .000 \\
\hline Hcy & $.232^{\star \star}$ & .000 \\
\hline ACR & $.179 \star \star$ & .003 \\
\hline eGFR & $-.434^{\star \star}$ & .000 \\
\hline MAU & $.248^{\star \star}$ & .000 \\
\hline$a-M G$ & $.354^{\star \star}$ & .000 \\
\hline Tf & $.303^{\star \star}$ & .000 \\
\hline $\lg$ & $.257^{\star \star}$ & .000 \\
\hline NEUT & $.221^{\star \star}$ & .000 \\
\hline LY & 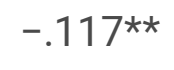 & .006 \\
\hline NLR & $.248^{\star \star}$ & .000 \\
\hline CRP & $.143^{\star \star}$ & .001 \\
\hline HOMAIR & $-.313^{\star \star}$ & .006 \\
\hline PFG & .037 & .398 \\
\hline FINS & $-.241^{\star}$ & .035 \\
\hline C-Peptide & -.022 & .727 \\
\hline 2hC-Peptide & -.086 & .167 \\
\hline $\mathrm{HbA1c}$ & .003 & .945 \\
\hline TCSS score & $.454^{\star \star}$ & .000 \\
\hline
\end{tabular}


** Significant correlation at .01 level; * significantly correlated at the .05 level.

Spearman correlation analysis (Table 4) showed that:a positive correlation (the correlation coefficient was positive) was found between the accumulation of skin AGEs and the risk scores of the four major complications of diabetes(CVD risk score, DPN risk score, DR risk score, and DN risk score), renal function indicators ( $\mathrm{Cr}$, CysC, Hcy, ACR, MAU, a-MG, Tf, and U-Ig G), inflammatory indicators (NEUT, NLR, and CRP), and TCSS score. However, a negative correlation was found with LY, HOMAIR, FINS and eGFR (the correlation coefficient was negative).

\title{
Correlation study between DPN grading and routine examination
}

\author{
Table 5
}

Correlation analysis of P. J. Dyck classification with vibration threshold, current threshold, and TCSS classification

\begin{tabular}{lllll}
\hline & & VPT & CPT & TCSS \\
\hline Grade of neuropathy & Correlation coefficient rs value & .475 & .235 & .565 \\
\hline & Sig. (double side) & $.000^{\star *}$ & $.000^{\star *}$ & $.000^{\star *}$ \\
\hline & $\mathrm{N}$ & 560 & 560 & 560
\end{tabular}

**. The correlation was significant when the confidence (double measure) was 0.01 .

Results (Table 5):P. J. Dyck grading was significantly correlated with vibration sensation threshold, current threshold and TCSS grading (all $P=0.000$ ), indicating that neuropathy of different degrees was correlated with the three examination grades. The correlation coefficient $\left(r_{s}\right)$ was between 0.2 and 0.6 , indicating that a positive correlation was found between DPN grading in this study and routine examination.

\section{Comparison of AGEs among groups in DPN grades}




\section{Table 6}

Comparison of AGEs cumulants among different DPN grades

\begin{tabular}{|c|c|c|c|c|c|c|}
\hline Group & Classification & $\mathrm{N}$ & mean & Standard deviation & $\mathrm{F}$ & $P$ \\
\hline \multirow[t]{3}{*}{ VPT } & $<15$ & 229 & 81.05 & 14.067 & \multirow[t]{3}{*}{26.335} & \multirow[t]{3}{*}{$0.000 *$} \\
\hline & $15-25$ & 207 & 85.38 & 16.099 & & \\
\hline & $>25$ & 124 & 93.65 & 17.067 & & \\
\hline \multirow[t]{3}{*}{ CPT } & Vormal & 327 & 83.36 & 13.467 & \multirow[t]{3}{*}{8.717} & \multirow[t]{3}{*}{$0.000 *$} \\
\hline & Less & 196 & 89.28 & 18.624 & & \\
\hline & Allergy & 37 & 83.35 & 20.996 & & \\
\hline \multirow[t]{4}{*}{ TCSS } & Normal & 78 & 77.86 & 13.886 & \multirow[t]{4}{*}{35.426} & \multirow[t]{4}{*}{$0.000 *$} \\
\hline & Mild & 339 & 82.64 & 13.515 & & \\
\hline & Moderate & 136 & 95.95 & 18.087 & & \\
\hline & Severe & 7 & 99.54 & 20.24 & & \\
\hline
\end{tabular}

*The significance level is 0.05 .

One-way ANOVA (Table 6): Within the classification of VPT, CPT, and TCSS, significant differences in AGEs were found between groups (all $P<0.05$ ). The linear and non-weighted significance of VPT and TCSS groups is all $<0.05$, indicating a linear relationship between the differences.

\section{ROC curve}

Figure 4 shows that the area under the curve of AGEs cumulant and neuropathy risk value is greater than 0.5 , indicating that both AGEs cumulant and neuropathy risk value have certain accuracy in diagnosing DPN. 
Table 7

Area under the curve

\begin{tabular}{|c|c|c|c|c|c|}
\hline \multirow[t]{3}{*}{ Test variable } & \multirow[t]{3}{*}{ AUC } & \multirow[t]{3}{*}{$S E^{a}$} & \multirow[t]{3}{*}{ Asymptotic sig. } & \multicolumn{2}{|l|}{$95 \% \mathrm{Cl}$} \\
\hline & & & & Lower bound & Upper \\
\hline & & & & & bound \\
\hline AGEs & .769 & .029 & .000 & .712 & .826 \\
\hline Neuropathy risk score & .743 & .028 & .000 & .688 & .799 \\
\hline
\end{tabular}

Test variable: AGE, neuropathy scores had at least one knot between positive and negative actual status groups. Statistics can be biased. a: under nonparametric assumptions; b: null hypothesis: real area $=0.5$.

In Table, significance $P=0.000<0.05$, the area under the curve of AGEs cumulative volume and neuropathy risk value was 0.769 and 0.743 , respectively. The confidence intervals were $(71.2 \%-82.6 \%)$ and $(68.8 \%-79.9 \%)$, respectively, indicating that both of them were significant in the diagnosis of DPN, and the accumulative value of AGEs was more valuable in the diagnosis of DPN than the risk value of neuropathy.

Table 8

Curvilinear coordinates

\begin{tabular}{llclll}
\hline Test variable & $\begin{array}{l}\text { Positive if greater than or } \\
\text { equal to }{ }^{\text {a }}\end{array}$ & Sensitivity & $\begin{array}{l}1- \\
\text { Specificity }\end{array}$ & Specificity & $\begin{array}{l}\text { Youden } \\
\text { index }\end{array}$ \\
\hline AGEs cumulants & 77.65 & .731 & .291 & 0.709 & 0.440 \\
\hline $\begin{array}{l}\text { Neuropathy risk } \\
\text { score }\end{array}$ & 66.2500 & .676 & .291 & 0.709 & 0.385 \\
\hline
\end{tabular}

Test variable : AGE, neuropathy scores had at least one knot between positive and negative actual status groups. a: The minimum boundary value is the minimum observation and test value minus 1 , and the maximum boundary value is the maximum observation and test value plus 1 . All other boundary values are the average of two adjacent observational test values.

See the curve coordinates to get Table 8: The maximum Youden's index of AGEs cumulant was 0.440, and the corresponding AGEs cumulant value was 77.65, which could be used as the critical value of DPN diagnosis. The corresponding sensitivity and specificity were 0.731 and 0.709 , respectively. Similarly, the maximum Youden's index of neuropathy risk value was 0.385 , and the corresponding neuropathy risk value was 66.25 , which could be used as the critical value of DPN diagnosis. The corresponding sensitivity and the specificity were 0.676 and 0.709 , respectively.

\section{Discussion}

\section{AGEs and DPN}


The pathogenesis of DPN has been recognized as follows. First, the polyol pathway: in the environment of high blood glucose level, aldose reductase activity is abnormally increased, and sorbitol production is obviously increased. Because of its poor permeability, it remained in the cell, leading to hypertonic edema, and affecting $\mathrm{Na}^{+}-\mathrm{k}^{+}$ATPase ase activity. Second, oxidative stress and inflammation: oxidative stress induces the generation of reactive oxygen species, lipid peroxidation, and even DNA damage in the body. On the one hand, the ability of the human body to scavenge free radicals is weakened; on the other hand, the production of free radicals increases, and peripheral nerve oxidative damage can cause excessive excitement of nerve conduction, abnormal sensation, and even pain ${ }^{[14]}$. Moreover, the lack of neurotrophic factors can directly or indirectly affect the normal structure and function of the neurons. PKC is involved in the activation of key regulatory proteins responsible for neural function and neurotransmitter synthesis. The activation of the abovementioned mechanisms can increase oxidative stress, which in turn can promote the formation of glycation products, and the formation cycle continues to deteriorate.

Additionally, new research findings are emerging. F. Shimizu believed that AGEs decreased the production of tight junction protein - 5 (claudin-5) in PnMECs by increasing the autocrine signal of vascular endothelial growth factor secreted by PnMEC and negatively regulated fibronectin, collagen type IV, and timp- 1 by transforming the release of growth factor (TGF)-beta ${ }^{[15]}$, thereby causing basement membrane hypertrophy and disrupting the blood-nerve barrier. Other studies suggest that AGEs can inhibit and impair the function of Schwann cells by promoting the release of inflammatory cytokines, affecting their reproduction, migration, and apoptosis ${ }^{[16]}$. AGEs can also modify nerve proteins so that the nerves do not work properly. The peripheral nerve myelin sheath modified by AGEs is easily engulfed by macrophages, which stimulates macrophages to secrete protease, which may lead to demyelination of diabetic neuropathy ${ }^{[17]}$. Glycosylation may alter the structure and functional properties of the axons, leading to atrophy and degradation of the axons and slowing down the conduction function of the axons.

The first experimental report in $2004{ }^{[18]}$ proposed that skin fluorescence as a noninvasive evaluation method was associated with AGEs values obtained from skin biopsies of patients with diabetes and healthy controls. This method was also recognized as the skin fluorescence ability to reflect AGE levels and was the basis of subsequent studies. However, until now, studies on peripheral neuropathy and AGEs of T2DM are few. Karabouta $Z$ et al. ${ }^{[19]}$ have confirmed that minors with T2DM are different from those with T1DM. Patients with T2DM can develop peripheral neuropathy soon after diagnosis. Thus, a simple and feasible method (indicator) is required to effectively screen such populations and can be used as an indicator of treatment or follow-up.

Ye Chengsong et al. ${ }^{[20]}$ confirmed that compared with the normal group, the skin AGEs and serum AGEs detected by fluorescence spectrometry were significantly higher in diabetic patients, indicating that the skin fluorescence AGEs test had the following advantages: noninvasive, sensitive, and high specificity. The Chinese Academy of Sciences' successful use of this spectral technique is achieved by using fullwavelength reflectance to correct the interference caused by differences in subjects' skin color. The result is superior to the international use of ultraviolet reflectance only. This has laid a solid foundation for the 
noninvasive detection and promotion of glycation products. DM-Scan, developed and designed, is noninvasive, fast, easy to operate, repeatable, and accurate. The technology and equipment are suitable for rapid risk screening and follow-up of diabetes and complications in developing countries with large populations.

\section{Discussion of research results}

\section{Differences of various indexes among different DPN grades Comparison of AGEs between different DPN grades}

It is found in this study (Tables 1 and 2, Fig. 2) that with the increase of the severity of DPN, the accumulations of AGEs and the risk scores of the four major complications showed an increasing trend, indicating that all of them had certain reference significance for grading the severity of DPN lesions. It can also be inferred that the four major complications of diabetes have the same pathogenesis. In addition, Additionally, no significant difference was found between grades 2 and 3 of AGEs in this study, which may be related to the inconsistency of sample size, and may also indicate that its specificity in grade 2 and 3 is weak, so further studies are needed in the future.

\section{Comparison of general data among different DPN grades}

Homocysteine (Hcy) may stimulate the production of oxygen free radicals through a large amount of accumulation in the body, which may lead to the emergence and aggravation of oxidative stress, reduce the production of NO, and damage the vascular endothelium. In turn, it creates conditions for the accumulation of lipid plaques and the formation of thrombosis, and accelerates the ischemia and hypoxia of microvessels and peripheral nerve tissues. At the same time, it can directly kill neurons, damage the nucleus, mitochondria and other. Cystatin $\mathrm{C}$ is a link in the process of Hcy increase. It can also induce inflammation and affect arterial endothelium and function, thereby indirectly affecting nerve tissue $^{[21]}$. The results of this experiment show that a significant difference was observed between DPN and $\mathrm{Cys} C$ in varying degrees. However, they need deeper basic experimental demonstration in the aspects of generation and pathogenic mechanism.

DPN has been proven to be a chronic inflammatory state, and low levels of inflammation play a special role in T2DM and its complications, and inflammation is correlated with vascular endothelial dysfunction and blood cell coagulation. Traditional inflammatory indicators including white blood cell count can predict the pathological condition of chronic inflammation to a large extent. CRP can lead to the increase of vascular endothelial factor, leading to the thickening of the basilar membrane of small vessels, and blood circulation disorder. Moreover, the immune function of CRP promotes the increase of white blood cells, which stimulates the activation of complement at the same time, and finally leads to cell apoptosis $^{[22]}$. The NLR is a novel marker reflecting a variety of chronic inflammatory diseases, which can reflect congenital and adaptive immune responses. Lymphocytes are often used as primers as a response indicator of systemic inflammation. Some studies suggest that NLR has a specific effect on DPN for 
studying inflammatory factors ${ }^{[23]}$. Based on the results of this study, it can be confirmed to some extent that NLR has a certain significance in the diagnosis of DPN, whereas CRP only has a difference between DPN grades 0 and 3 , so NLR can be speculated to play a more important role in the future chronic inflammatory diseases.

This study found that besides the eGFR, the aggravated pathological changes gradually reduced, and the severity of renal function indexes with DPN has a roughly growing trend, which once again proved that DPN is associated with the pathogenesis of diabetes small vascular lesions. However, the kidney function index can be a reference value to the diagnosis of DPN, and expanding the sample for further studies is necessary.

The main function of MAPK is to ensure the energy stability of tissue cells, which can be activated and promoted when energy is insufficient. Increased intracellular $\mathrm{Ca}^{2+}$ can increase AMPK activity and enhance autophagy function ${ }^{[24]}$. Related literature has also shown that small-fiber neuropathy is prone to G856D mutation. Nav1.7 ion channel was excessively opened, and the axis showed time-dependent degeneration, which was associated with increased intracellular calcium ion content and decreased ATP level ${ }^{[25]}$. However, this study found that no significant statistical difference was found between the calcium ion concentration and DPN lesion grading, but there was a tendency to decrease step by step.

C-peptide in T2DM has pro-inflammatory and pro-sclerosing effects, which may be caused by different levels of insulin, c-peptide, and insulin resistance (IR), as well as different receptors and signaling pathways ${ }^{[26]}$. Additionally, C-peptide has been proven in recent years to cause regeneration of small fibers and reduce the necrosis of nerves and vascular endothelium, which may be related to the enhancement of $\mathrm{Na}^{+}-\mathrm{K}^{+}$-ATPase activity and the promotion of $\mathrm{NO}$ release ${ }^{[27]}$. A cross-sectional study has shown that IR often plays a special role in the course of DPN in patients with metabolic syndrome ${ }^{[28]}$. A Korean study ${ }^{[29]}$ concluded that IR was independently correlated with neuropathy in patients with T2DM. In this study, no significant differences were found between the groups in C-peptide and HOMA-IR, which may be because most of the patients in this study were in the late stage of T2DM, the pancreatic islet function was generally poor, and sufficient sample size was not included.

Studies have found that low-density lipoprotein in diabetic patients is easily affected by glycation, forming AGEs-LDL, which slows down the metabolism of LDL-C, reduces the clearance, and makes it more difficult to reduce blood lipid. After phagocytosis in the body, it is easy to form complexes that gather on the vascular endothelium and cross-link with each other, and may cause severe inflammation through NF-KB factors, eventually leading to vascular lesion and neuropathy ${ }^{[30]}$. In this study, no significant correlation was found between LDL-C, TC, and other lipid indicators and DPN, which may be related to the long-term adherence to diet education of diabetic patients in our hospital department and the active clinical intervention of lipids.

\section{Correlation analysis of skin AGEs accumulations and other indicators}


Correlation analysis (Tables 3 and 4) shows that the accumulation of skin AGEs was significantly correlated with multiple indicators. Among them, the inflammatory indicators (lymphocyte, middle fluid ratio, and CRP), islet function (insulin 1-2 h after meal), renal function-related indicators (eGFR, Cr, CysC, Hcy, ACR, urinary microalbumin, a microglobulin, and urinary transferrin, urinary immunoglobulin), and TCSS score were closely related to DPN. On the one hand, the occurrence of DPN is related to inflammation and decreased islet function, and DPN and diabetic nephropathy may have the same pathological mechanism. On the other hand, it also indicates that interoperability may exist between these indicators and AGEs. Thus, combining AGEs with these indicators was speculated to improve the detection rate of DPN and better evaluate the severity of DPN.

\section{Correlation study between DPN classification and other examination grades}

Presently, the international standard for grading DPN has the highest recognition, the earliest use, and the most extensive use, which is based on the classification standard for diabetic neuropathy of P. J. Dyck. However, in recent years, the severity of DPN has been gradually graded by VPT, current threshold (CPT), and related scores including the TCSS score, which has also achieved good recognition.

This test for P. J. Dyck classification standard, together with the above three kinds of classification methods, adopted the correlation analysis (Table 5). The results showed a good correlation $(P=0.000<$ 0.01), with the correlation coefficient ( $\mathrm{rs}$ ) between 0.2 and 0.6.The P.J. Dyck classification standard and the degree of the vibration sense threshold of neuropathy, current threshold level and grade of TCSS also showed a lot of good positive correlations between the classification methods. All four methods could well reflect the severity of DPN.

\section{Discussion on the diagnostic value of skin AGEs cumulant to DPN}

\section{AGEs were compared among groups with different DPN grades}

As can be seen from Table 6 and the broken line statistics (Fig. 3), with the increase of VPT, the accumulations of AGEs gradually increased. The cumulative amount of AGEs in the CPT normal group was smaller than that in the weakened group. TCSS showed that the cumulative amount of AGEs increased with the increase of the degree (however, in this study, TCSS judged that the heavy group had little relationship with other groups, which might be related to the small sample size of this group). This study has confirmed that with the increase of DPN, the accumulations of AGEs also show an increasing trend. In the four DPN grading methods mentioned above, the accumulations of AGEs gradually increase with the increasing degree of DPN. In this study, a lot of positive correlation was found between the grading standard of P. J. Dyck neuropathy and the three grading methods of vibration sensation 
threshold degree, current threshold degree, and TCSS grading. Thus, the accumulation of skin AGEs was inferred to evaluate and predict the grading of DPN and its severity.

\section{ROC curve}

According to the ROC curve (Fig. 4, Table 7), the area under the curves of AGEs cumulative volume and neuropathy risk value were 0.769 and 0.743 , respectively, both of which were larger than 0.5 , indicating that both AGE cumulative volume and neuropathy risk value had certain accuracy in the diagnosis of DPN. According to the curve coordinates (Table 8), the maximum approximate rank index of AGEs cumulant was 0.440 , and the corresponding AGEs cumulant value was 77.65 , which could be used as the critical value of DPN diagnosis. The corresponding sensitivity and specificity were 0.731 and 0.709 , respectively. Similarly, the maximum approximate rank index of neuropathy risk value was 0.385 , and the corresponding neuropathy risk value was 66.25 , which could be used as the critical value of DPN diagnosis. The corresponding sensitivity and specificity were 0.676 and 0.709 , respectively. Therefore, both the accumulative value of AGEs and the risk value of neuropathy can be used to diagnose DPN, and the accumulative value of AGEs is relatively higher. Both results were obtained by skin AGE detection system for detection and evaluation, so AGE Pro is speculated to screen DPN better..

The noninvasive detection system of glycosylated products used in this study was provided by Hefei Institutes of Physical Sciences, Chinese Academy of Sciences, model DM-Scan. AGE Pro, according to specific emission wavelength fluorescence intensity and the amount of which accumulated in the skin of the principle of positive correlation, in safe light triggers the human skin, through the whole wavelength reflectance correction subjects including skin color difference of interference (different from foreign countries, UV reflectivity is used for correction, and the correction effect is better, and more suitable for yellow race). Based on the optical signal response subjects which accumulate in the body, and in combination with the built-in relevant model (with a variety of diabetes complications, oxidative stress, and aging), the risk assessment of diabetes and its complications, detection of long-term control of diabetes, and assessment of oxidative stress and human aging can be achieved.

\section{Conclusion}

The cumulative amount of skin AGEs can be used as the diagnostic index and the prediction and evaluation index of DPN. With high sensitivity, high specificity, and economic and social value, AGE Pro can be used as a reliable detection tool and be popularized.

\section{Limitations And Prospects}

This study has several limitations. First, this study is mainly a retrospective observation. Because of time constraints and other reasons, a longitudinal observation was not possible. Second, although diagnostic criteria and exclusion criteria were limited for the included cases, AGEs could not be affected by food, cooking methods, related drugs, and other factors. 
For the abovementioned limitations, the following methods can be adopted for improvement: First, dynamically track the changes of patients' AGEs in treatment, and deeply discuss the mechanism between AGEs and diseases. Next, conduct a detailed investigation report and guidance of diet and treatment for DPN patients and analyze the roles of influential factors of AGEs in the occurrence and development of diseases.

\section{Abbreviations}

UA: Blood uric acid; TC: Cholesterol; WBC: White blood cell; TG: Triglyceride; Cr: Serum creatinine; ACR: Albumin-Creatinine-Ratio; eGFR: Glomerular filtration rate ; MAU: Microscale albuminuria;a-MG: aMicroglobulin; Tf: Transferrin; U-Ig G: Urinary immune globin; NEUT: Neutrophil count; LY: Lymphogranulocyte count; NLR: Neutrolphil to lymphocyte ratio; CRP: C-reactive protein; HOMA-IR: Homeostasis model assessment-Insulin resistance; Course: Course of disease; SBP: Systolic pressure; DBP: Diastolic pressure; CVD: Cardio-cerebrovascular disease; R:Diabetic retinopathy; DN: Diabetic nephropathy; BMI: Body mass index;HbA1c: Haemoglobin A1c; HDL: High-density lipoprotein cholesterol; LDL: Lowdensity lipoprotein cholesterol; BUN: Blood urea nitrogen; Cys-C: Cystatin-C; HCY: Homocysteinel; FBG: Fasting blood glucose;1hPBG:1-h postprandial blood glucose; 2hPBG:2-h postprandial blood glucose; 3hPBG:3-h postprandial blood glucose; INS: insulin; INS60':60minutes postprandial insulin;INS120':120minutes postprandial insulin; INS180':180minutes postprandial insulin; $1 \mathrm{~h}$ C-Peptide:1-h standardized postprandial C-peptide; $2 \mathrm{~h}$ C-peptide: 2-h standardized postprandial C-peptide;3h C-Peptide:3-h standardized postprandial C-peptide.

\section{Declarations}

\section{Acknowledgments}

We sincerely thank Chengdu University of Traditional Chinese Medicine for the support of statistical methods and English correction. Thanks for the support of Sichuan Provincial Science and Technology Planning Project

(19ZDYF1497).

\section{Authors' contributions}

XWZ and QC conceived and designed the research. XWZ performed most of the experiments. SWZ and WXY performed parts of the experiments.WXY and SWZ analyzed the data. XWZ and QC wrote the manuscript. All data were generated in-house. All authors agree to be held accountable for all aspects of the research and confirm that the data are accurate. And all authors have read and approved the manuscript. 


\section{Funding}

This work was supported by Sichuan Provincial Science and Technology Planning Project (19ZDYF1497). The funding body did not influence the study design, data collection, analysis, and publication.

\section{Availability of data and materials}

The datasets used and/or analysed during the current study are available from the corresponding author on reasonable request.

\section{Ethics approval and consent to participate}

The study protocol is in accordance with the ethical guidelines of the 1975 Declaration of Helsinki and has been approved by the ethics committees at the Hospital of Chengdu University of Traditional Chinese Medicine (No.2017KL-033). All the participants signed the informed consent in writing prior to inclusion in the study, and the access and use of electronic medical records were approved by the Hospital of Chengdu University of Traditional Chinese Medicine.

\section{Consent for publication}

Not applicable.

\section{Competing interests}

The authors declare that they have no conflict of interest to disclose.

\section{References}

1. Thornalley Paul-J. Glycation in diabetic neuropathy: characteristics, consequences, causes, and therapeutic options.[J]. Int. Rev. Neurobiol., 2002, 50: 37-57.

2. Vincent Andrea-M.,Russell James-W.,Low Phillip. Oxidative stress in the pathogenesis of diabetic neuropathy[J]. Endocrine Reviews, 2004, 25(4): 612-628.

3. Yagihashi Soroku,Yamagishi Shin-Ichiro,Wada Ryuichi. Pathology and pathogenetic mechanisms of diabetic neuropathy: correlation with clinical signs and symptoms.[J]. Diabetes Res. Clin. Pract., 2007: 0-9.

4. Nakamura J,Kato K,Hamada Y. A protein kinase C-beta-selective inhibitor ameliorates neural dysfunction in streptozotocin-induced diabetic rats.[J]. Diabetes, 1999, 48(10): 5-2090. 
5. Aleksandra araszkiewicz1 Agnieszka-Gandecka1-Michał-Nowicki2. Association between small fiber neuropathy[J]. ORIGINAL ARTICLE, 2016, 126(11): 847-853.

6. A Di-Pino,W Currenti,F Urbano. High intake of dietary advanced glycation end-products is associated with increased[J]. Nutrition, metabolism, and cardiovascular diseases: NMCD, 2017, 27(11): 978984.

7. Monica Negrean,Alin Stirban,Bernd Stratmann. Effects of low- and high-advanced glycation endproduct meals onmacro- and microvascular endothelial function and oxidative stressin patients with type 2 diabetes mellitus[J]. The American journal of clinical nutrition, 2007, 85(5): 43-1236.

8. Rajaobelina K,Farges B,Nov S. Skin autofluorescence and peripheral neuropathy four years later in type 1 diabetes [J]. Diabetes/Metabolism Research and Reviews, 2017, 33(2): 0-0.

9. Baqiyyah n. conway PHD.,Stephen fernandez MPH.,Vanita r. aroda MD. Skin Intrinsic Fluorescence CorrelatesWith Autonomic and Distal SymmetricalPolyneuropathy in Individuals WithType 1 Diabetes[J]. DIABETES CARE, 2011, 34(4): 1000-1005.

10. P.j. dyck MD,Kratz K.M.,Lehman K.A. The Rochester Diabetic Neuropathy Study: Design, criteria for types of neuropathy, selection bias, and reproducibility of neuropathic tests [J]. Neurology, 1991, 41(6): 799-807.

11. Liu P. Application of TCSS score in screening and treatment of peripheral neuropathy in type 2 diabetes mellitus [J]. Hainan Medical Journal, 2012, (17): 21-23.

12. Investigation group on chronic complications of diabetes of Chinese diabetes association. Retrospective investigation and analysis of chronic complications and related risk factors of inpatients with diabetes in China for 10 years. [J]. Chinese Journal of Diabetes, 2003, (4): 5-10.

13. Chinese diabetes association. Chinese guidelines for the prevention and treatment of type 2 diabetes (2013 edition) [J]. Chinese journal of diabetes, 2014: 2-42.

14. Ayodeji babatunde oyenihi,Ademola olabode ayeleso,Emmanuel mukwevho. Antioxidant strategies in the management of diabetic neuropathy.[J]. Biomed Res Int, 2015: 515042.

15. F. shimizu,Y. sano,H. haruki. Advanced glycation end-products induce basementmembrane hypertrophy in endoneurial microvesselsand disrupt the blood-nerve barrier by stimulatingthe release of TGF- $\beta$ and vascular endothelialgrowth factor (VEGF) by pericytes[J]. Diabetologia, 2011, 54(6): 1517-1526.

16. Zhang $\mathrm{M}$, Zhang $\mathrm{HL}$. Effect of glycation end products on in vitro function of schwann cells and its mechanism [J]. Chinese General Practice, 2013, 16(19): 1729-1732.

17. H Vlassara,M Brownlee,A Cerami. Recognition and uptake of human diabetic peripheral nerve myelin by macrophages[J]. Diabetes, 1985, 34(6): 7-553.

18. R meerwaldt,R graaff,Ph oomen. Simple non-invasive assessment of advanced glycation endproductaccumulation[J]. Diabetologia, 2004, 47(7): 30-1324.

19. Karabouta Z,Barnett S,Shield J. Peripheral neuropathy is an early complicationof type 2 diabetes in adolescence[J]. Pediatric diabetes, 2008, 9(2): 4-110. 
20. Ye CS,Wang YK,Ye SD. Correlation study of skin AGEs and serum AGEs [J]. Chinese Journal of Laboratory Diagnosis, 2014, 18(11): 1768-1770.

21. Chen FL,Wang WH,Chen M. Role of plasma homocysteine and cystatin $\mathrm{C}$ in diabetic peripheral neuropathy [J]. Chinese Journal of Gerontology, 2010, 30(1): 19-22.

22. Zhou Y,Xian LH. Research progress on early diagnosis of diabetic peripheral neuropathy by serum cystatin C and c-reactive protein [J]. Modern Medicine \& Health, 2018, 34(9): 1363-1365.

23. Liu SY,Zheng HP,Zhu XM. Neutrophil-to-lymphocyte ratio is associated with diabetic peripheral neuropathy in type 2 diabetes patients.[J]. Elsevier B.V., 2017, 130: 90-97.

24. You chul chung,Ji hee lim,Oh Hyun-Mi. Calcimimetic restores diabetic peripheral neuropathy by ameliorating apoptosis and improving autophagy[J]. Cell Death \& Disease, 2018, 9: 3-18.

25. Harshvardhan rolyan,Shujun liu,Janneke gj hoeijmakers. A painful neuropathy-associated Nav1.7 mutant leads to time-dependent degeneration of small-diameter axons associated with intracellular $\mathrm{Ca}^{2+}$ dysregulation and decrease in ATP levels[J]. SAGE Publications, 2016, 12: 173-188.

26. Dusica vasic,Daniel walcher,Fabrizio montecucco. C-Peptide: A New Mediator of Atherosclerosis in Diabetes[J]. Mediators of Inflammation, 2012, 2012: 1-8.

27. Zhao FF,An C,Wang Q. Correlation between peripheral neuropathy and c-peptide level in type 2 diabetes mellitus [J]. China Medicine and Pharmacy, 2013, 3(4): 20-21, 26.

28. Ling han,Lijin ji,Jing chang. Peripheral neuropathy is associated with insulin resistance independent of metabolic syndrome[J]. BioMed Central, 2015, 7(1): 1-6.

29. Kee ook lee,Ji sun nam,Chul woo ahn. Insulin resistance is independently associated with peripheraland autonomic neuropathy in Korean type 2 diabetic patients[J]. Acta Diabetologica, 2012, 49(2): 97-103.

30. Yang Q,Ma Q,Zeng QC. Induction and mechanism of AGE-LDL on calcification of human aortic valve interstitial cells and inflammatory response [J]. Shandong Medical Journal, 2018, 58(19): 22-26.

\section{Figures}



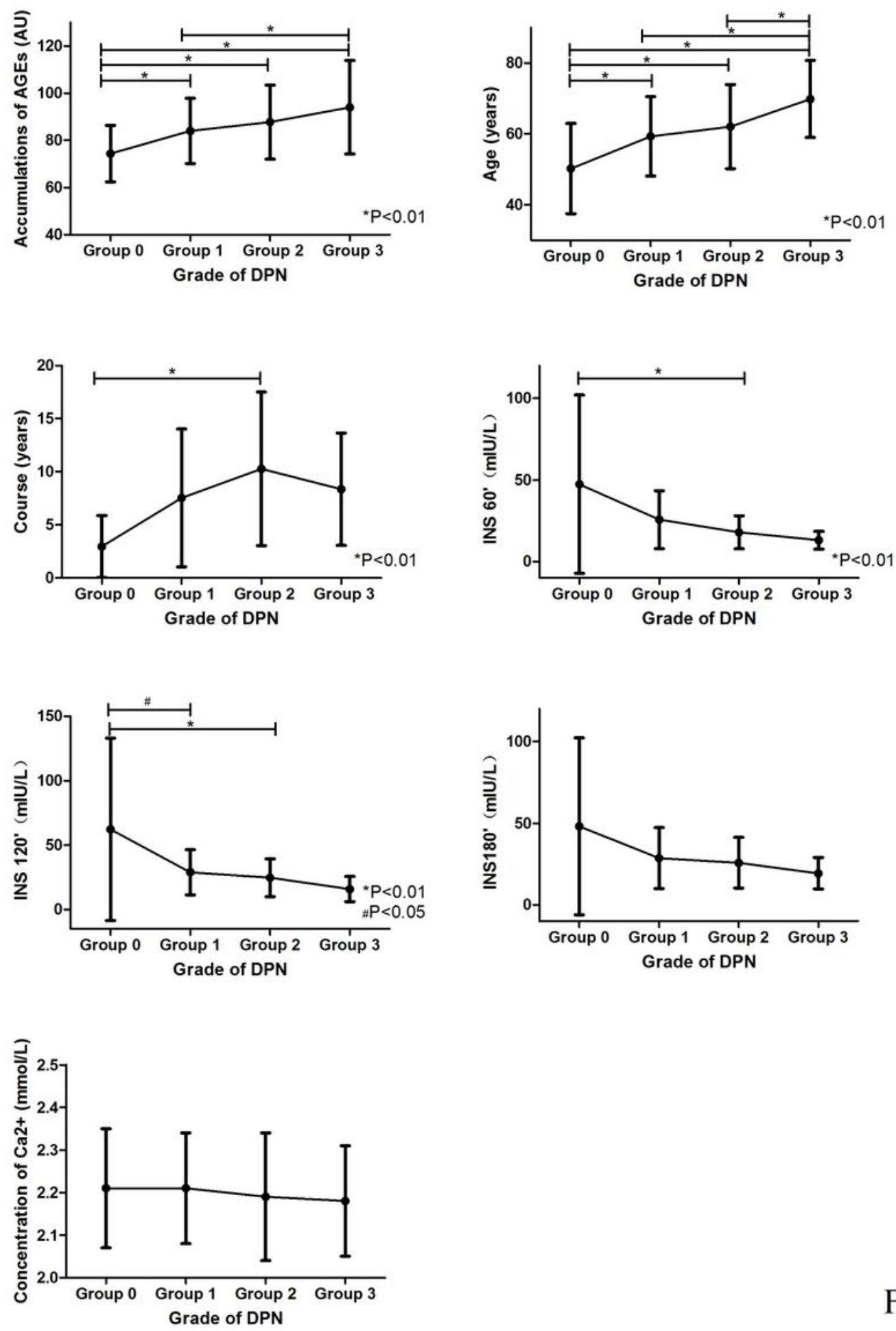

FIG 1

\section{Figure 1}

Results (FIG 1): $₫$ Comparison of AGEs between groups: significant differences $(P=0.000)$ of AGEs were found among grades $0,1,2$, and 3 of DPN, and significant differences $(P=0.000)$ of AGEs were found between grades 1 and 3 . $\otimes$ Comparison of general data between groups: significant differences in age were found among grades $0,1,2$, and 3 (all $P=0.000$ ), and between grades 1 and 3,2 and 3 (all $P=$ 0.000). The course of DPN grades 0 and 2 was significantly different $(P=0.007)$. Significant differences 
were also found between DPN grades 0 and grade 2 at $1 \mathrm{~h}$ postprandial insulin $(P=0.007)$, between grades 0 and 1 , and between grades 0 and 2 at $2 \mathrm{~h}$ postprandial insulin $(P=0.012, P=0.005)$. $\nabla$ With the increase of DPN severity, the accumulative AGEs, age, and course of diseases all showed an increasing trend. Insulin decreased after 1 and $2 \mathrm{~h}$ postprandial. Additionally, although no significant statistical difference was found in calcium ion concentration and insulin level at $3 \mathrm{~h}$ postprandial, both showed a gradually declining trend as can be seen from the statistical figures.
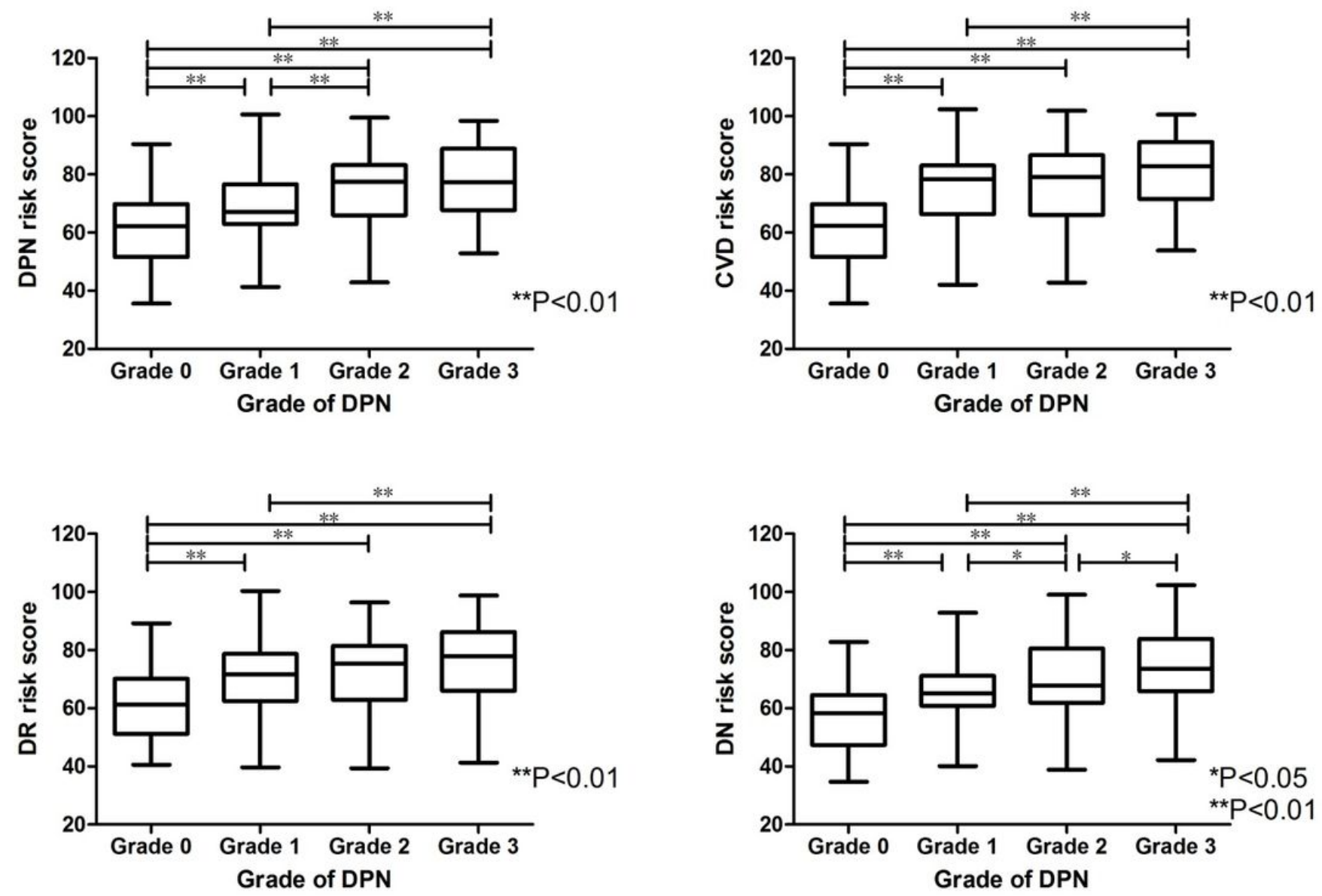

FIG 2

Figure 2

Four risk scores of diabetes complications in different DPN groups: There were significant differences in DPN risk score between grades $0,1,2$, and 3 , between grades 1,2 , and 3 , and between grades 2 and 3 ( $P<$ 0.01 or $P<0.05)$. Significant differences in CVD and DR risk score were found between grades $0,1,2$, and 3 , and between grades 1 and $3(P<0.01)$.Significant differences in $D N$ risk score were found between grades $0,1,2$, and 3 , between grades 1,2 , and $3(P<0.01)$. 

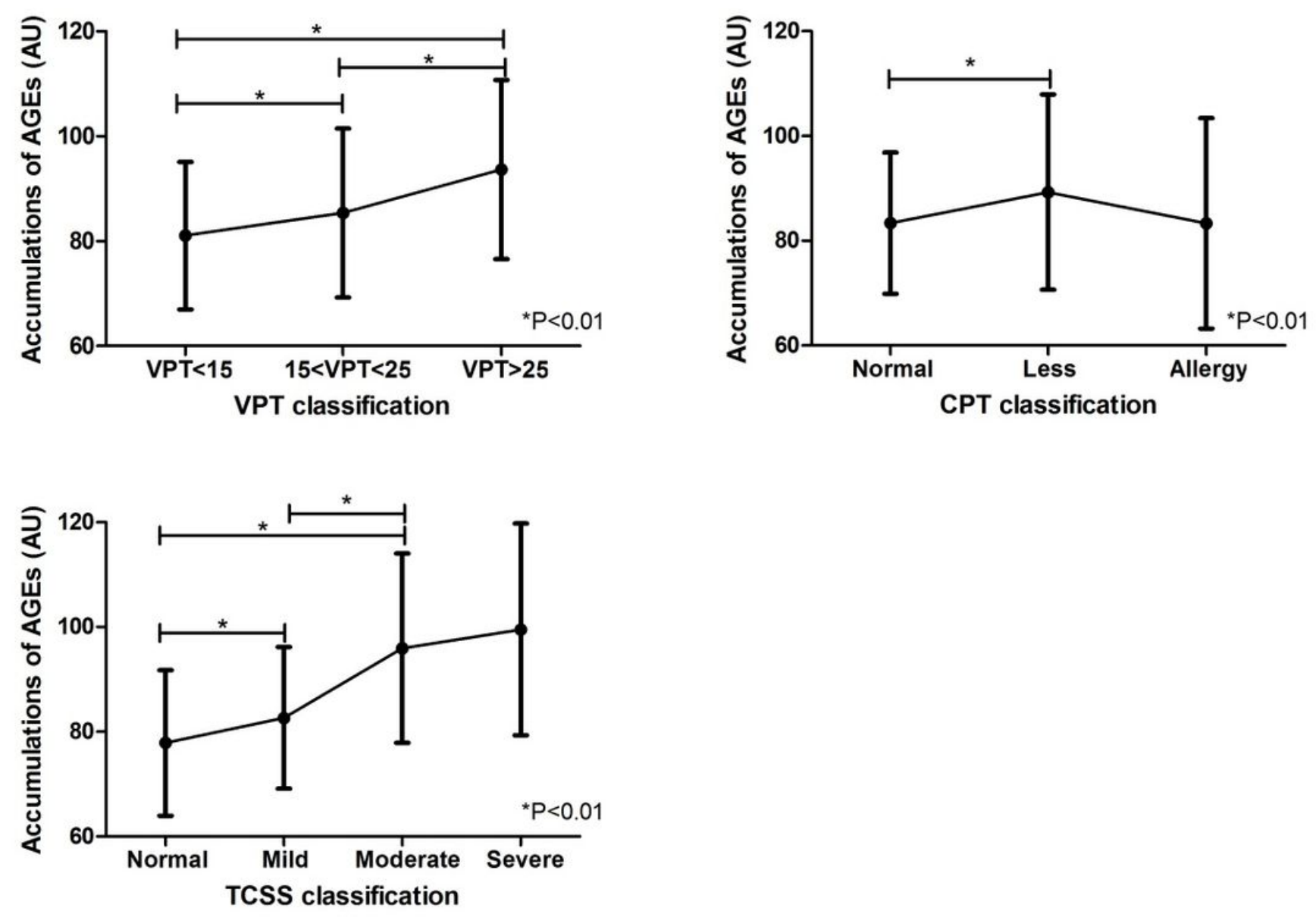

FIG 3

Figure 3

Multiple comparisons are shown as follows (FIG 3): With the increase of VPT, AGE accumulations increased gradually. Significant differences were found between VPT $<15$, VPT 15-25, and VPT $>25$ and between VPT 15-25 and VPT >25 ( $<<0.01)$. The cumulative amount of AGEs in the CPT normal group was significantly lower than that in the less group. A significant difference in AGEs was found between the CPT normal group and the less group $(P<0.01)$. With the increase of TCSS grading, the cumulative amount of AGEs increases, Significant differences were found between the TCSS normal group and those in the mild and moderate groups $(P<0.01$ or $P<0.05)$. The difference between the mild and moderate group was found to be significant $(P<0.01)$. 
ROC curve

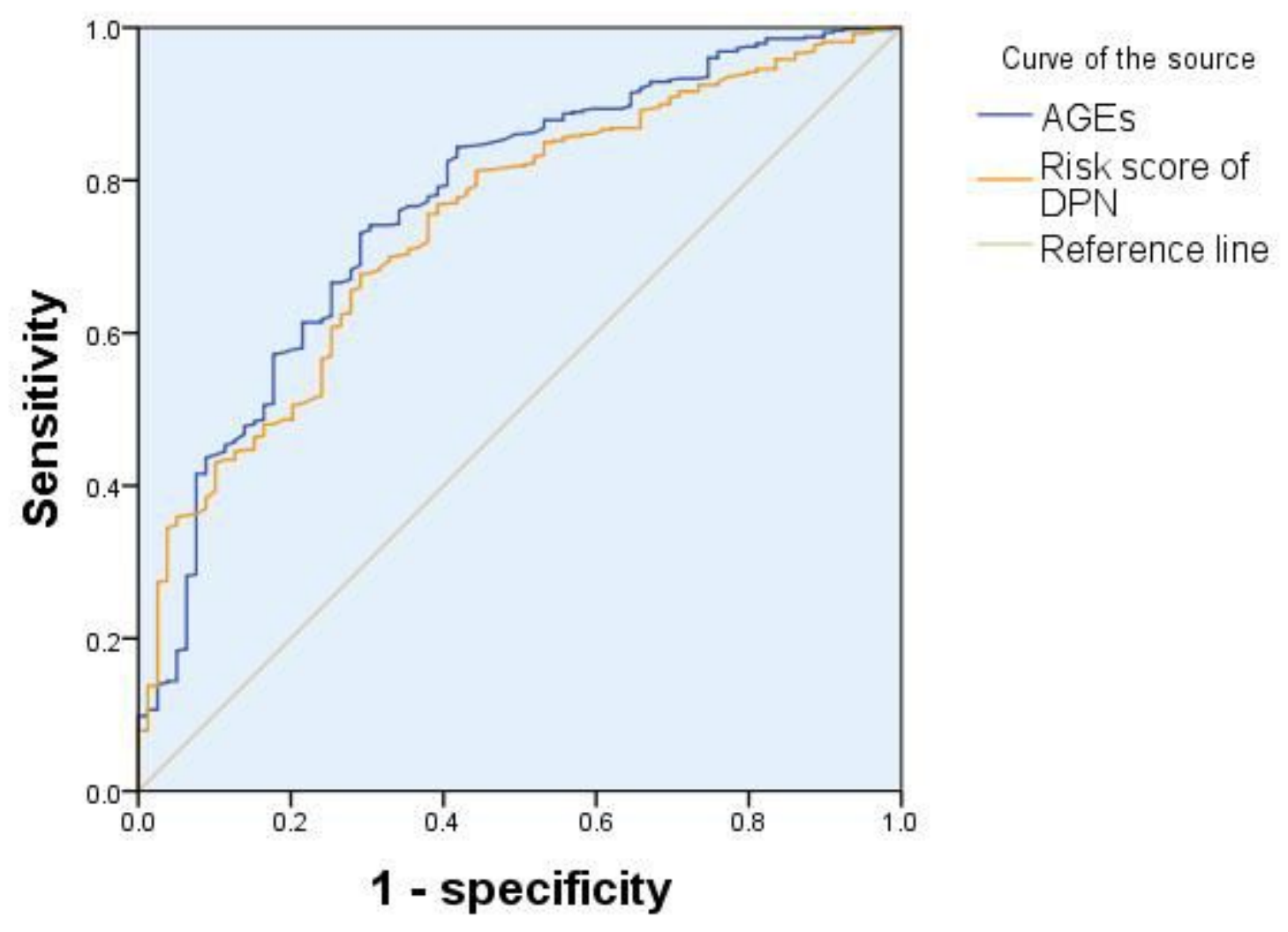

The diagonal segment generated by the junction. $\quad$ FIG 4

\section{Figure 4}

shows that the area under the curve of AGEs cumulant and neuropathy risk value is greater than 0.5 , indicating that both AGEs cumulant and neuropathy risk value have certain accuracy in diagnosing DPN.

\section{Supplementary Files}

This is a list of supplementary files associated with this preprint. Click to download.

- Figs.jpg 